\title{
Authentication analysis of cod liver oil from beef fat using fatty acid composition and FTIR spectra.
}

\begin{abstract}
This study aimed to authenticate cod liver oil (CLO) from beef fat (BF) by determining the level of BF as a fat adulterant in CLO. Two instrumental techniques, namely GC-FID for fatty acid analysis and Fourier transform infrared (FTIR) spectroscopy, were exploited for such authentication. The decreased level of some fatty acids, especially eicosapentaenoic acid (EPA) and docosahexaenoic acid (DHA), could be used as an indicative means to detect the adulteration of CLO with BF. In addition, FTIR spectroscopy combined with partial leastsquares (PLS) at frequency regions of $1200-1000 \mathrm{~cm} \mathrm{-1}$ was successfully developed for the quantification of BF in CLO. Using the PLS model, the errors obtained in calibration and prediction samples were $0.55 \%$ and $0.82 \% \mathrm{v} / \mathrm{v}$, respectively.
\end{abstract}

Keyword: Authenticity; Chemometrics; Gas chromatography; FTIR spectroscopy; Fats; Oils. 